\title{
Influence of a hole-transport layer on light-induced degradation of mixed organic-inorganic halide perovskite solar cells
}

Takeyuki Sekimoto, Taisuke Matsui, Takashi Nishihara, Ryusuke Uchida, Takashi Sekiguchi and Takayuki Negami Institute for Energy and Material/Food Resources, Technology Innovation Division, Panasonic Corporation, Osaka 570-8501, Japan.

E-mail: sekimoto.takeyuki@jp.panasonic.com

\section{MICROWAVE PHOTOCONDUCTIVITY DECAY MEASUREMENTS}

Microwave photoconductivity decay ( $\mu$-PCD) measurements were performed in air at room temperature using an LTA-1800SP system (Kobelco Research Institute) with laser $(\lambda=349 \mathrm{~nm}$, pulse width $=$ $5 \mathrm{~ns}$ ) and microwave (frequency $=26 \mathrm{GHz}$ ) sources.

The $\mu$-PCD technique has been widely applied to various semiconductors, such as silicon, ${ }^{1-3}$ amorphous indium gallium zinc oxide, ${ }^{4}$ and barium silicide. ${ }^{5}$ In $\mu$-PCD, the changes in the microwave reflectivity are detected when the sample is irradiated by pulsed laser light. The microwave reflectivity is proportional to the carrier concentration, and the carrier lifetime can be determined from the decay curve. To examine the damage of the organic-inorganic halide perovskite (OIHP) caused by the hole-transport layer (HTL), we applied the $\mu$-PCD technique to glass/OIHP and glass/OIHP/HTL (5 $\mathrm{nm})$ structures. The laser penetration depth $\left(l_{\mathrm{p}}\right)$ was approximately 10 to $100 \mathrm{~nm}$. The incident laser and microwave entered from the film side. The lack of a signal from the HTL film alone was checked in advance to confirm that information about the OIHP/HTL interface and OIHP bulk could be obtained for the OIHP/HTL structures. Typical decay curves are shown in Fig. S1a.

The peak value of the decay curve is proportional to $N_{0} \gamma$, where $N_{0}$ is the number of incident photons, and $\gamma$ ranges from 0.5 for radiative electron-hole recombination to 1.0 for non-radiative Shockley-ReadHall (SRH) recombination. ${ }^{4,6}$ The $N_{0}$ dependence of the peak values for OIHP thicknesses $(d)$ of 35, 105, 250, and $550 \mathrm{~nm}$ is shown in Fig. S1b. In the region with high $N_{0}\left(\geq 2.9 \times 10^{14} \mathrm{~cm}^{-2}\right), \gamma<0.5$, so we did not include the data in this range in the analysis. One possible reason for $\gamma<0.5$ is that the Auger recombination process may become dominant because of the influence of excess carrier generation. Conversely, in the region with low $N_{0}\left(\leq 2.9 \times 10^{13} \mathrm{~cm}^{-2}\right)$, all of the samples have $\gamma>0.5$ and $\gamma$ tends to decrease with $d$. Thus, to analyze the OIHP surface and OIHP/HTL interface, the $\mu$-PCD data with low $N_{0}$ were used.

Decay curves were fitted using three exponential terms: ${ }^{5} I(t)=$ $I_{1} \exp \left(-t / \tau_{\text {Auger }}\right)+I_{2} \exp \left(-t / \tau_{\text {SRH }}\right)+I_{3} \exp \left(-t / \tau_{\text {SRH-trapping }}\right)$, where $t$ is time. Similar to the literature, ${ }^{5}$ we hypothesized that the first term is attributed to Auger recombination, which is significantly suppressed because of the low $N_{0}$ of $2.9 \times 10^{12} \mathrm{~cm}^{-2}$ and low carrier concentration of the OIHP layer (typically $10^{15}-10^{16} \mathrm{~cm}^{-3}$ ). In addition, we hypothesized that the second and third terms are derived from SRH recombination processes without and with the carrier trapping effect, respectively. In the above equation, $I_{i}(i=1,2,3)$ is the coefficient, and

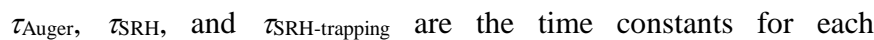
recombination process, where $\tau_{\text {Auger }}<\tau_{\text {SRH }}<\tau_{\text {SRH-trapping. Therefore, }}$ $\tau_{\mathrm{SRH}}$ in the second term was used to analyze the samples.

Plots of $1 / \tau_{\mathrm{SRH}}$ versus $1 / d$ are shown in Fig. S1c. We evaluated the bulk lifetime $\tau_{\mathrm{b}}$ and surface recombination velocity $S$ using the equation $1 / \tau_{\mathrm{SRH}}=1 / \tau_{\mathrm{b}}+2 S / d($ valid for $S<D /(4 d)),{ }^{1}$ where $D$ is the minority carrier diffusion constant. Assuming a carrier mobility $\mu=$ $\sim 20 \mathrm{~cm}^{2} \mathrm{~V}^{-1} \mathrm{~s}^{-1}$ (Ref. 7) and the Einstein relation $D=\mu k T / q$, where $k$ is the Boltzmann constant, $T$ is the absolute temperature, and $q$ is the elementary charge, respectively, the $D$ value is estimated to be $\sim 0.5$ $\mathrm{cm}^{2} \mathrm{~s}^{-1}$. More correct analysis requires that $d$ is comparable with or much smaller than $l_{\mathrm{p}}{ }^{2,6}$ Therefore, the fitting in Fig. S1c was performed in the range $d \leq 105 \mathrm{~nm}$. The $\tau_{\mathrm{b}}$ and $S$ values are summarized in Table $\mathrm{S} 1$ along with some literature data. ${ }^{7-11}$ The $S$ values of our samples are much lower than $D /(4 d)$. The OIHP film in this study has $S=2.6 \mathrm{~cm} \mathrm{~s}^{-1}$, which is lower than the order of about $10 \mathrm{~cm} \mathrm{~s}^{-1}$ for an unpassivated OIHP film ${ }^{7}$ and was close to the $S$ value of $2 \mathrm{~cm} / \mathrm{s}$ for an amorphous silicon/crystalline silicon interface in a silicon heterojunction solar cell, ${ }^{3}$ verifying that the OIHP films with device-grade quality have comparably low $S$. The $\tau_{\mathrm{b}}$ value of $451 \mathrm{~ns}$ for the OIHP film is close to the reported values of $\sim 477$ (Ref. 8) and $\sim 511 \mathrm{~ns}^{7}$ Using the equation $L=\left(D \tau_{\mathrm{b}}\right)^{1 / 2}$, the diffusion length of minority carriers $(L)$ is $\sim 5 \mu \mathrm{m}$, which is sufficiently larger than $d$. Importantly, by coating HTL on the OIHP film, $\tau_{\mathrm{b}}$ decreases to $230 \mathrm{~ns}$ for the OIHP/PTAA structure and $412 \mathrm{~ns}$ for the OIHP/Spiro structure. Moreover, $S$ increases to $8.9 \mathrm{~cm} \mathrm{~s}^{-1}$ for the OIHP/PTAA structure and $3.3 \mathrm{~cm} \mathrm{~s}^{-1}$ for the OIHP/Spiro structure. These results provide quantitative evidence that the PTAA coating forms a poorer interface with OIHP compared with the Spiro coating and promotes LID of the OIHP bulk in the vicinity of the OIHP/PTAA interface.

\section{REFERENCES}

(1) Arafat, Y.; Mohammedy, F. M.; Hassan, M. M. S. Optical and Other Measurement Techniques of Carrier Lifetime in Semiconductors. Int. J. Optoelec. Eng., 2012, 2, 5-11.

(2) Schüler, N.; Anger, S.; Dornich, K.; Niklas, J. R.; Bothe, K. Limitations in the accuracy of photoconductance-based lifetime measurements. Sol. Energy Mat. Sol. Cells, 2012, 98, 245-252.

(3) Taguchi, M.; Yano, A.; Tohoda, S.; Matsuyama, K.; Nakamura, Y.; Nishiwaki, T.; Fujita, K.; Maruyama, E. 24.7\% Record Efficiency HIT Solar Cell on Thin Silicon Wafer. IEEE J. Photovoltaics, 2014, 4, 96-99.

(4) Lee, D. H.; Kawamura, K.; Nomura, K.; Yanagi, H.; Kamiya, T.; Hirano, M.; Hosono, H. Steady-state photoconductivity of amorphous In-Ga-Zn-O. Thin Solid Films, 2010, 518, 3000-3003.

(5) Bayu, M. E.; Trinh, C. T.; Takabe, R.; Yachi, S.; Toko, K.; Usami, N.; Suemasu, T. Minority-carrier lifetime and photoresponse properties of $\mathrm{B}$-doped $\mathrm{p}-\mathrm{BaSi}_{2}$, a potential light absorber for solar cells. Jpn. J. Appl. Phys., 2017, 56, 05DB01.

(6) Chen, Y.; Yi, H. T.; Wu, X.; Haroldson, R.; Gartstein, Y. N.; Rodionov, Y. I.; Tikhonov, K. S.; Zakhidov, A.; Zhu, X.-Y.; Podzorov, V. Extended carrier lifetimes and diffusion in hybrid perovskites revealed by Hall effect and photoconductivity measurements. Nat. Commun., 2016, 7, 12253.

(7) Staub, F.; Hempel, H.; Hebig, J.-C.; Mock, J.; Paetzold, U. W.; Rau, U.; Unold, T.; Kirchartz, T. Beyond Bulk Lifetimes: Insights into Lead Halide Perovskite Films from Time-Resolved Photoluminescence. Phys. Rev. Appl., 2016, 6, 044017. 
(8) Yang, Y.; Yang, M.; Moore, D. T.; Yan, Y.; Miller, E. M.; Zhu, K.; Beard, M. C. Top and bottom surfaces limit carrier lifetime in lead iodide perovskite films. Nat. Energy, 2017, 2 16207.

(9) Yang, Y.; Yan, Y.; Yang, M.; Choi, S.; Zhu, K.; Luther, J. M.; Beard, M. C. Low surface recombination velocity in solutiongrown $\mathrm{CH}_{3} \mathrm{NH}_{3} \mathrm{PbBr}_{3}$ perovskite single crystal. Nat. Commun., 2015, 6, 7961.

(10) Fang, H.-H.; Adjokatse, S.; Wei, H.; Yang, J.; Blake, G. R.; Huang, J.; Even, J.; Loi, M. A. Ultrahigh sensitivity of methylammonium lead tribromide perovskite single crystals to environmental gases. Sci. Adv., 2016, 2, e1600534.

(11) Brenes, R.; Guo, D.; Osherov, A.; Noel, N. K.; Eames, C.; Hutter, E. M.; Pathak, S. K.; Niroui, F.; Friend, R. H.; Islam, M. S.; Snaith, H. J.; Bulovic, V.; Savenije, T. J.; Stranks, S. D. Metal Halide Perovskite Polycrystalline Films Exhibiting Properties of Single Crystals. Joule, 2017, 1, 155-167.
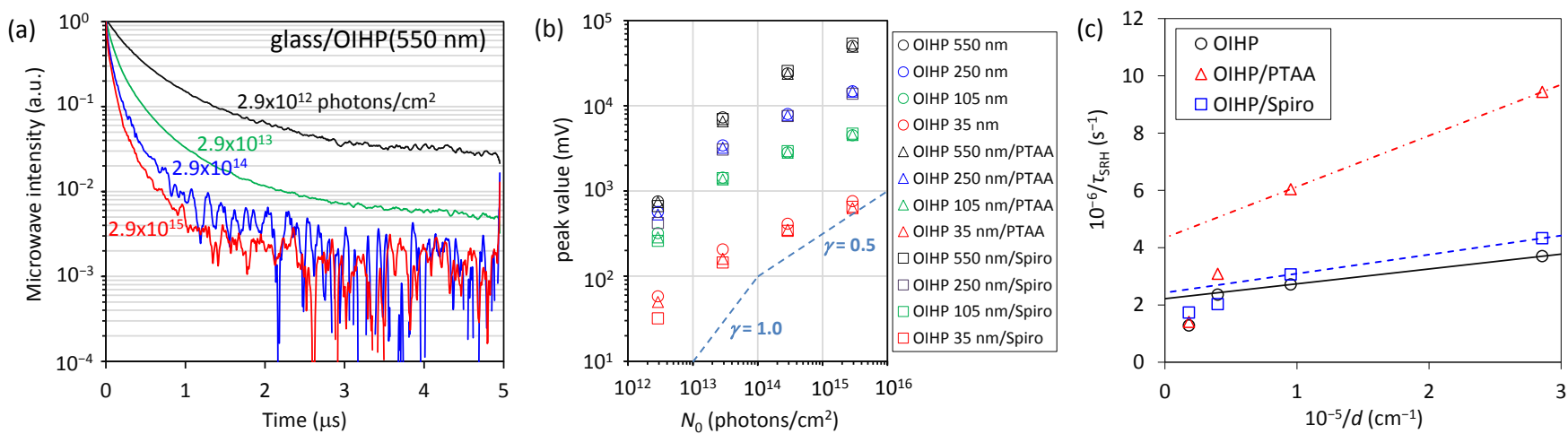

Figure S1. (a) Normalized $\mu$-PCD decay curves of OIHP films with different $N_{0}$. (b) $N_{0}$ dependence of the peak value. The dashed lines represent the cases of $\gamma=0.5$ and 1.0. (c) Plots of $1 / \tau$ SRH versus $1 / d$.

Table S1. Comparison of the bulk lifetime $\left(\tau_{\mathrm{b}}\right)$ and surface recombination velocity $(S)$ values of the samples with reported values. ${ }^{a}$ Ref. 8, ${ }^{b}$ Ref. 9, ${ }^{c}$ Ref. 10, ${ }^{d}$ Ref. 7, ${ }^{e}$ Ref. 11.

\begin{tabular}{lcc}
\hline Sample & $\tau_{\mathrm{b}}(\mathrm{ns})$ & $S(\mathrm{~cm} / \mathrm{s})$ \\
\hline OIHP & 451 & 2.6 \\
OIHP/PTAA 5nm & 230 & 8.9 \\
OIHP/Spiro 5nm & 412 & 3.3 \\
Unpassivated OIHP single crystal & $1600^{a}$ & $\sim 2800^{a}, 3400^{b}$ \\
Passivated OIHP single crystal & $4500^{c}$ & $\sim 3^{c}$ \\
Unpassivated OIHP thin film & $\sim 477^{a}, \sim 511^{d}$ & $\sim 450^{a}, \sim 10^{d}$ \\
Passivated OIHP thin film & & $\sim 0.4^{e}$ \\
\hline
\end{tabular}

